\title{
On the interaction of mesoscopic quantum systems with gravity
}

\author{
Claus Kiefer* and Carsten Weber \\ Institut für Theoretische Physik, Universität zu Köln, Zülpicher Str. 77, 50937 Köln, Germany
}

\begin{abstract}
Key words quantum gravity, mesoscopic systems, gravitational waves, quantum fluids, gravitoelectromagnetism.

PACS 04.60.-m, 04.80.Nn, 72.20.-i

We review the different aspects of the interaction of mesoscopic quantum systems with gravitational fields. We first discuss briefly the foundations of general relativity and quantum mechanics. Then, we consider the non-relativistic expansions of the Klein-Gordon and Dirac equations in the post-Newtonian approximation. After a short overview of classical gravitational waves, we discuss two proposed interaction mechanisms: (i) the use of quantum fluids as generator and/or detector of gravitational waves in the laboratory, and (ii) the inclusion of gravitomagnetic fields in the study of the properties of rotating superconductors. The foundations of the proposed experiments are explained and evaluated.
\end{abstract}

\section{Quantum theory and the gravitational field}

In this introductory section we shall give a brief review of the relation between quantum theory and gravity. For more details and references we refer to [1].

The gravitational interaction is distinguished by the fact that it interacts universally with all forms of energy. It dominates on large scales (relevant for cosmology) and for compact objects (such as neutron stars and black holes). All presently known features of gravitational physics are successfully described by the theory of general relativity (GR), accomplished by Albert Einstein in 1915. ${ }^{1}$ In this theory, gravity is not interpreted as a force acting on space and in time, but as a representation of the geometry of spacetime. This is a consequence of the equivalence principle stating the (local) equivalence of free fall with the gravity-free case. Mass generates curvature which in turn acts back on the mass. Curvature can also exist in vacuum; for example, it can propagate with the speed of light in the form of gravitational waves. In contrast to other physical theories, spacetime in GR plays a dynamical role and not the role of a rigid background structure.

Experimentally, GR is a very successful theory [4]. Particularly impressive examples are the observations of the double pulsar PSR $1913+16$ by which the existence of gravitational waves has been verified indirectly. Many interferometers on Earth are now in operation with the goal of direct observations of these waves. This would open a new window to the universe ('gravitational-wave astronomy'). After 40 years of preparation, the ambitious satellite project Gravity-Probe B was launched in April 2004 in order to observe the 'Thirring-Lense effect' predicted by GR. This effect states that a rotating mass (here the Earth) forces local inertial systems in its neighbourhood to rotate with respect to far-away objects. GR has also entered everyday life in the form of the global positioning system GPS; without the implementation of GR, inaccuracies in the order of kilometres would easily arise over the time span of days [5]. A recent survey of GR tests in space can be found in [6] and the references therein.

\footnotetext{
* Corresponding author: e-mail: kiefer@thp.uni-koeln.de

${ }^{1}$ Exceptions may be the Pioneer anomaly [2] and the rotation curves of galaxies [3], which could in principle demand a more general theory. This is unclear at the moment.
} 
GR can be defined by the 'Einstein-Hilbert action',

$$
S_{\mathrm{EH}}=\frac{c^{4}}{16 \pi G} \int_{\mathcal{M}} \mathrm{d}^{4} x \sqrt{-g}(R-2 \Lambda),
$$

where $c, G$, and $\Lambda$ are the speed of light, the gravitational constant, and the cosmological constant, respectively; $g$ denotes the determinant of the four-dimensional metric and $R$ the Ricci scalar. The integration is over a four-dimensional manifold $\mathcal{M}$, and a surface term has been omitted for simplicity. In addition one has an action, $S_{\mathrm{m}}$, describing non-gravitational fields minimally coupled to the gravitational field. From it one can derive the energy-momentum tensor

$$
T_{\mu \nu}=\frac{2}{\sqrt{-g}} \frac{\delta S_{\mathrm{m}}}{\delta g^{\mu \nu}},
$$

which acts as a 'source' for the gravitational field. The variation of $S_{\mathrm{EH}}+S_{\mathrm{m}}$ leads to Einstein's field equations,

$$
G_{\mu \nu} \equiv R_{\mu \nu}-\frac{1}{2} g_{\mu \nu} R+\Lambda g_{\mu \nu}=\frac{8 \pi G}{c^{4}} T_{\mu \nu} .
$$

From general features ('singularity theorems') one can see that GR specifies-in contrast to Newtonian gravity ${ }^{2}$-its own limits: there are situations where GR can no longer be valid. Typically, this happens inside black holes and near the Big Bang. The general belief is that only the inclusion of quantum theory can describe such situations.

Quantum theory is of a universal nature, too. In contrast to GR, however, it gives the general frame for theories describing particular interactions. In fact, all known interactions besides gravity (that is, the strong and electro-weak interactions) are successfully described by quantum theory. At the heart of quantum theory is the superposition principle, which is experimentally well established and which is crucial for many modern developments such as quantum information theory, cf. [7].

A consistent application of the quantum framework to the gravitational field is referred to as quantum gravity. The construction of such a theory is one of the big open problems in theoretical physics. What are the main motivations for the search of such a theory?

- All non-gravitational interactions are described by quantum theories. Since gravity interacts universally with all quantum fields, it is natural to expect that it is described by a quantum theory as well. The prevailing idea of unification of all interactions gives an argument for the existence of a unified quantum theory including gravity.

- The origin of the universe and the evaporation of black holes cannot be understood within the classical theory. Cosmology and black-hole physics are thus incomplete without quantum gravity.

- Ordinary (non-gravitational) quantum theory and GR contain concepts which are incompatible: whereas quantum theory uses a rigid background structure (Newton's absolute time $t$ in quantum mechanics, or Minkowski spacetime in quantum field theory or-in situations where this approximation holds-in relativistic quantum mechanics), spacetime in GR is dynamical. This is sometimes called the problem of time. There should therefore exist an encompassing theory of quantum gravity in which these concepts are modified. In fact, the straightforward quantization of GR leads to the result that no external time can exist at all at the most fundamental level.

At present, there exist two main classes of approaches in quantizing gravity [1]. One is quantum GR, which is obtained by the application of quantization rules to GR. Among these, the path-integral method

\footnotetext{
2 Note, however, that Newtonian cosmology leads to intrinsic inconsistencies.
} 
and canonical quantization are especially noteworthy. Preliminary results seem to indicate a discrete structure of spacetime at the fundamental level and an avoidance of the classical singularities. The alternative to this class is superstring theory ('M-theory'). In contrast to quantum GR, it aims at a unified quantum theory of all interactions with gravity. Quantum gravity then follows for sufficiently low energies when the gravitational interaction can be considered as a separate entity. Superstring theory also seems to indicate a discrete structure of spacetime. It predicts the presence of effective terms violating the equivalence principle. This could be subject to experimental tests [8]. In addition, one can consider effective theories of quantum gravity, which are valid only for low enough energies. They may be either derived from a fundamental theory or from heuristic considerations. From such effective theories one can derive, for example, quantum-gravitational correction terms to the Newtonian potential [1].

The fundamental scale of quantum gravity is the Planck scale. It is obtained by combining the speed of light $(c)$, the gravitational constant $(G)$, and the quantum of action $(\hbar)$ into units of length, time, and mass. They read explicitly

$$
\begin{aligned}
l_{\mathrm{P}} & =\sqrt{\frac{\hbar G}{c^{3}}} \approx 1.62 \times 10^{-33} \mathrm{~cm}, \\
t_{\mathrm{P}} & =\frac{l_{\mathrm{P}}}{c}=\sqrt{\frac{\hbar G}{c^{5}}} \approx 5.40 \times 10^{-44} \mathrm{~s}, \\
m_{\mathrm{P}} & =\frac{\hbar}{l_{\mathrm{P}}}=\sqrt{\frac{\hbar c}{G}} \approx 2.17 \times 10^{-5} \mathrm{~g} \approx 1.22 \times 10^{19} \mathrm{GeV} .
\end{aligned}
$$

It is believed that it is the smallness (or largeness) of these Planck units that has hitherto prevented a direct experimental access to test theories of quantum gravity.

Historically, an attempt to combine ordinary quantum theory with classical gravity in a consistent way has led to the formulation of the so-called 'semiclassical Einstein equations', in which the expectation value of the operator-valued energy-momentum tensor with respect to some quantum state is coupled to the classical gravitational field. Instead of (3) one would then have

$$
R_{\mu \nu}-\frac{1}{2} g_{\mu \nu} R+\Lambda g_{\mu \nu}=\frac{8 \pi G}{c^{4}}\left\langle\Psi\left|\hat{T}_{\mu \nu}\right| \Psi\right\rangle
$$

However, it is now generally believed that these equations can at best be an approximation. First, a 'Schrödinger cat-type experiment' performed by Page and Geilker [9] has given results in contradiction to 77. These authors have considered a box in which there are two rigidly connected masses and a quantummechanical system that is in a superposition of 'being decayed' and 'being non-decayed'. In case of a decay, a mechanism is put into work that destroys the rigid connection of the masses and enables them to approach each other. The superposition of the quantum-mechanical system is then transferred to the masses. Outside the box is a Cavendish balance. According to (7), the balance would follow the expectation value of the mass distribution and slowly swing in time. The experiment, however, shows an abrupt deflection occurring at a certain time, in conflict with (7).

The second point concerns the investigation of models describing the coupling of a quantum system to a classical system ('hybrid models'), which seems to lead to inconsistencies. Equation (7) usually holds if the mean square deviation ('fluctuation') of $\hat{T}_{\mu \nu}$ is small. This condition is violated in the Page-Geilker experiment. It is also violated in another interesting situation - the gravitational radiation emitted by quantum systems, as discussed by Kuo and Ford [10]. There the predictions of a linearized theory of quantum gravity differ drastically from (7). Another case where the fluctuations of the energy-momentum tensor are large occurs when $\left\langle\Psi\left|\hat{T}_{\mu \nu}\right| \Psi\right\rangle$ can become negative. This happens, for example, in the Casimir effect or the case of a squeezed vacuum ('particle creation') relevant in cosmology or black-hole physics.

This study is devoted to the interaction of mesoscopic quantum systems (including for definiteness also microscopic quantum systems) with gravity. The main motivations are the following: 
- As far as the relation of quantum theory with gravity is concerned, this is the only level where experiments have been either performed or have been suggested. A clear understanding of this interaction would thus be crucial for the development of quantum gravity. It could, in particular, help to obtain conceptual clarity. Moreover, approaches to quantum gravity suggest that there may be effects (such as the above-mentioned violation of the equivalence principle from string theory) that could be observed in this regime.

- It is of interest to study the realizability of experimental proposals connected with current theoretical work. This could lead to a decision about ideas predicting the observability of surprising effects. This will be discussed in detail in Sects. 3 and 4.

- This interaction can be used as a tool for other experiments. One example is the planned ESA satellite mission HYPER. It will use atom interferometry to test the equivalence principle and to measure the Thirring-Lense effect, which causes a phase shift in the interferometer, in its full dynamical evolution.

A deep understanding of this topic could build, on the way towards quantum gravity, a bridge to the next level of interaction between quantum theory and gravity - the level of quantum field theory in curved spacetime. Here, there exists a definite prediction: black holes radiate with a thermal spectrum ('Hawking effect'). In the special case of spherical symmetry and absence of charges ('Schwarzschild black hole'), this temperature is given by [11]

$$
T_{\mathrm{BH}}=\frac{\hbar c^{3}}{8 \pi k_{\mathrm{B}} G M} \approx 6.17 \times 10^{-8}\left(\frac{M_{\odot}}{M}\right) \mathrm{K},
$$

where $k_{B}$ denotes the Boltzmann constant and $M$ the mass of the black hole. This temperature is tiny and has not yet been observed. It is only of detectable size for sufficiently small black holes, which can only form in the very early universe. No such 'primordial black holes' have, however, hitherto been seen. There exists an effect in flat spacetime that is analogous to the Hawking effect. It arises for an observer who moves with constant acceleration through Minkowski space. Such an observer experiences the standard Minkowski vacuum as being filled with a thermal distribution of particles, for which the temperature is given by [12]

$$
T_{\mathrm{DU}}=\frac{\hbar a}{2 \pi k_{\mathrm{B}} c} \approx 4.05 \times 10^{-23} a\left[\frac{\mathrm{cm}}{\mathrm{s}^{2}}\right] \mathrm{K},
$$

where $a$ denotes the acceleration. This temperature is often referred to as the 'Davies-Unruh temperature'. There are proposals (and attempts) to observe this effect in particle accelerators or with ultra-intense lasers, cf. [13]. Of interest are also suggestions to study formal analogues of these effects with features from condensed matter physics such as Bose-Einstein condensation, cf. [14] and the references therein.

For definiteness, we restrict ourselves in this study to GR and standard quantum theory (plus unifications of both), and leave aside alternative theories of gravity as well as, for example, non-linear generalizations of quantum theory. Among such more general frameworks are the suggestions that the gravitational field might be the cause for a collapse of the wave function, cf. [15] and Chap. 8 in [7]. This would signal a breakdown of unitarity at the most fundamental level. There exist experimental proposals for a test of such ideas. For example, the interaction of one photon with a tiny mirror could lead to a superposition of mirror states, invoking in this process about $10^{14}$ atoms [16]. The hope then will be that one may be able to distinguish between standard environmental decoherence and a fundamental gravity-induced collapse. At the moment, however, one is still very far away from an experimental test.

As for alternatives to classical GR, there are different options. On the one hand, one may add additional fields in the gravitational sector, such as scalar fields. This leads to the Jordan-Brans-Dicke theory and generalizations thereof [17]. Typically, such theories lead to a spatiotemporal variation of coupling constants, from which one gets strong constraints on them. They play a role mainly as effective low-energy 
theories resulting from fundamental theories such as string theory. On the other hand, one may consider gauge theories of gravity. The simplest example is the Einstein-Cartan theory, which invokes the concept of a non-vanishing spacetime torsion (in addition to curvature), see, for example, [18] for a lucid introduction. In this theory, vierbein (instead of metric) and connection arise as the gauge potentials belonging to translations and Lorentz transformations, respectively. The corresponding gauge field strengths are torsion and curvature, respectively. The presence of torsion is needed in order to accomodate spin (for scalars and photons, the Einstein-Cartan theory is indistinguishable from GR). An even more general framework allows the presence of non-metricity, leading to metric-affine gauge theories of gravity, cf. [19]. As we shall mention below, the effects of torsion turn out to be negligible on laboratory scales (they may, however, play a role in the early universe), so we can safely restrict ourselves to GR for the purpose of this study.

\section{Quantum mechanics in external electromagnetic and gravitational fields}

In order to study the interaction of quantum systems with gravity, an appropriate formalism has to be developed. Since we are dealing here with situations where quantum field-theoretic effects such as particle creation can be neglected, it is sufficient to consider quantum mechanics in an external gravitational field. In the non-relativistic limit, the appropriate setting is the Schrödinger equation with an external gravitational field. Since some experiments are also sensitive to relativistic corrections, we discuss in the following the relativistic quantum-mechanical equations (Klein-Gordon and Dirac equation, respectively) and perform a non-relativistic expansion. The Klein-Gordon equation describes spinless particles (e.g. $\pi$-mesons), while the Dirac equation describes spin-1/2 particles. For most applications, the latter is the appropriate framework.

\subsection{The Klein-Gordon equation and its non-relativistic expansion}

The Klein-Gordon equation in flat spacetime without external fields is given by

$$
\left(\hbar^{2} \square-m^{2} c^{2}\right) \varphi\left(x^{\mu}\right)=0,
$$

where $m$ is the mass of the particle, and

$$
\square \equiv-\frac{\partial^{2}}{c^{2} \partial t^{2}}+\Delta \equiv \eta^{\mu \nu} \partial_{\mu} \partial_{\nu}
$$

The coupling to an external gravitational and electromagnetic field is achieved through 'minimal coupling', that is, through the substitution of the partial derivative $\partial_{\mu}$ by the covariant derivative $D_{\mu}$, which is defined by its action on a vector field $v^{\mu}$ as

$$
D_{\mu} v^{\nu}=\partial_{\mu} v^{\nu}+\Gamma_{\mu \lambda}^{\nu} v^{\lambda}-\frac{\mathrm{i} e}{\hbar c} A_{\mu} v^{\nu},
$$

where $\Gamma_{\mu \lambda}^{\nu}$ denotes the Christoffel symbol, $A_{\mu}$ the electromagnetic vector potential, and $e$ denotes the charge of the particle. Therefore, instead of (10) one has

$$
\left(\hbar^{2} g^{\mu \nu} D_{\mu} D_{\nu}-m^{2} c^{2}\right) \varphi\left(x^{\mu}\right)=0 .
$$

We assume the external fields to be sufficiently weak and slowly varying in space and time, in order to have no quantum field-theoretic effects. The Klein-Gordon equation (12) is then interpreted as a quantummechanical equation. For the purposes of this study it is appropriate to perform a non-relativistic approximation, that is, an approximation in powers of $c^{-2}$. This can technically be achieved by a FoldyWouthuysen-type of transformation or by an expansion of the phase of $\varphi$. Effectively, the formalism yields 
a separation into 'positive' and 'negative' energies. For the case of a pure electromagnetic field, the approximation scheme can be found, for example, in [20], and for the presence of both external fields in [21]. The result is a Schrödinger equation

$$
\mathrm{i} \hbar \frac{\partial}{\partial t} \psi(\mathbf{x}, t)=\tilde{H}_{\mathrm{eff}} \psi(\mathbf{x}, t)
$$

where $\psi$ denotes a redefined positive part of $\varphi$, and $\tilde{H}_{\text {eff }}$ is the non-relativistic Hamilton operator plus relativistic correction terms.

Since the external gravitational field is assumed to be weak, a post-Newtonian approximation should be sufficient [21]. A post-Newtonian approximation is an expansion in powers of the small parameter

$$
\frac{\bar{v}}{c} \sim\left(\frac{G \bar{M}}{c^{2} \bar{r}}\right)^{1 / 2}
$$

where $\bar{v}, \bar{r}$, and $\bar{M}$ are typical values for the velocities, radii, and masses of the involved bodies, respectively, and the virial theorem has been used. Gravitational radiation is not included in this approximation. One can then choose for the metric coefficients the expressions

$$
\begin{aligned}
& g_{00} \approx-\left(1-\frac{2 U}{c^{2}}+\frac{2 U^{2}}{c^{4}}\right), \\
& g_{0 i} \approx 0, \\
& g_{i j} \approx\left(1+\frac{2 U}{c^{2}}\right) \delta_{i j},
\end{aligned}
$$

where

$$
U(\mathbf{x}, t)=G \int \frac{\rho_{\mathrm{m}}\left(\mathbf{x}^{\prime}, t\right)}{\left|\mathbf{x}-\mathbf{x}^{\prime}\right|} \mathrm{d}^{3} x^{\prime}
$$

is the Newtonian potential corresponding to a mass density $\rho_{\mathrm{m}}\left(\mathbf{x}^{\prime}, t\right)$. Since the off-diagonal elements of the metric are neglected, rotation effects cannot be seen in this approximation. Since the Klein-Gordon equation (12) leads to the conservation of a scalar product different from that of the Schrödinger equation, the standard inner product

$$
\langle\psi \mid \chi\rangle=\int \mathrm{d}^{3} x \psi^{*} \chi
$$

is not conserved with respect to the Hamiltonian $\tilde{H}_{\text {eff }}$ in 13 . Conservation can only be obtained after a time-dependent transformation of the Hamiltonian and the wave function. After such a transformation has been performed, the effective Hamiltonian reads [21]

$$
\begin{aligned}
H_{\mathrm{eff}}= & m c^{2}-\frac{\hbar^{2}}{2 m} \Delta-e A_{0}-m U-\frac{\hbar^{4}}{8 m^{3} c^{2}} \Delta^{2} \\
& +\frac{3 \hbar^{2}}{2 m c^{2}}(U \Delta+\nabla U \cdot \nabla)+\frac{m U^{2}}{2 c^{2}}+\frac{3 \hbar^{2} \Delta U}{4 m c^{2}} \\
& -\frac{e}{4 m c}\left[1+\frac{\hbar^{2}}{2 m^{2} c^{2}} \Delta-\frac{3 U}{c^{2}}, \mathbf{B}\left(\mathbf{x}_{\mathrm{a}}, t\right) \mathbf{L}\right]_{+} \\
& -\mathbf{d}\left(\mathbf{E}_{\mathrm{T}}\left(\mathbf{x}_{\mathrm{a}}, t\right)+\frac{1}{c} \mathbf{v}_{\mathrm{a}} \times \mathbf{B}\left(\mathbf{x}_{\mathrm{a}}, t\right)\right)+\mathcal{O}\left(\frac{1}{c^{4}}\right) .
\end{aligned}
$$

Here, $\mathbf{x}_{\mathrm{a}}$ is the expectation value of the position operator (describing, for example, the position of an atom), $\mathbf{L}$ the angular momentum operator, $\mathbf{d}$ the electric dipole operator, and $\mathbf{v}_{\mathrm{a}}=\mathrm{d} \mathbf{x}_{\mathrm{a}} / \mathrm{d} t ; \mathbf{E}_{\mathrm{T}}$ and $\mathbf{B}$ denote the 
transversal part of the electric and magnetic field, respectively. The Hamiltonian (17) is Hermitian with respect to the scalar product (16).

The first four terms represent the rest energy, the non-relativistic kinetic term, and the electric and gravitational potentials, respectively, while the fifth term is the first relativistic correction to the kinetic term. The next four terms are relativistic corrections for the gravitational field. The term proportional to $\Delta U$ in (17) can be interpreted as a 'gravitational Darwin term', in analogy to the usual electric Darwin term $\propto \nabla \cdot \mathbf{E}$ arising from 'zitterbewegung' (the coordinate of a particle is smeared out over a length $\approx \hbar / m c$ ). The latter appears for the Klein-Gordon equation first at order $c^{-4}$. The remaining terms contain the usual terms for the electric and magnetic field as well as a commutator term between the magnetic field and the gravitational potential. Thus, one recognizes that the interaction of the matter field with the magnetic field is modified by the gravitational potential $U$, whereas its coupling to the transversal electric field and to $A_{0}$ remains unchanged in this order of approximation (compared to the gravity-free case). This is important for atom interferometry.

\subsection{The Dirac equation and its non-relativistic expansion}

The non-relativistic expansion of the Dirac equation in an external electromagnetic field can be treated by a Foldy-Wouthuysen transformation [20]. In this way one finds correction terms analogous to the electromagnetic terms in (17) but augmented by terms involving spin. They usually find applications in atomic physics (modification of the spectral lines found from the Schrödinger equation). Similarly, one can discuss the Dirac equation for rotating and accelerating systems and, correspondingly, for the case of an external gravitational field. For rotation $\boldsymbol{\omega}$ and acceleration a, the non-relativistic expansion has been performed in [22], while in [23], an exact non-relativistic result has been obtained for static spacetimes.

The Dirac equation in Minkowski space (and for Cartesian coordinates) reads

$$
\left(\mathrm{i} \gamma^{\mu} \partial_{\mu}+\frac{m c}{\hbar}\right) \psi(x)=0
$$

where $\psi(x)$ is a Dirac spinor, and

$$
\left[\gamma^{\mu}, \gamma^{\nu}\right]_{+} \equiv \gamma^{\mu} \gamma^{\nu}+\gamma^{\nu} \gamma^{\mu}=2 \eta^{\mu \nu}
$$

The transformation into an accelerated frame is achieved by replacing partial derivatives with covariant derivatives, see, for example, [24],

$$
\partial_{\mu} \longrightarrow D_{\mu} \equiv \partial_{\mu}+\frac{\mathrm{i}}{2} \sigma^{m k} \Gamma_{\mu m k},
$$

where $\sigma^{m k}=\mathrm{i}\left[\gamma^{m}, \gamma^{k}\right]$ is the generator of the Lorentz group, and $\Gamma_{\mu m k}$ denotes the components of the connection. Latin indices here denote anholonomic components, that is, components that are not derivable from a coordinate basis. From the equivalence principle, one would expect that the form in an accelerated frame gives also the appropriate form in curved space-time, where

$$
\left[\gamma^{\mu}, \gamma^{\nu}\right]_{+}=2 g^{\mu \nu}
$$

For the formulation of the Dirac equation in curved space-time, one has to use the tetrad ('vierbein') formalism, in which a basis $e_{n}=\left\{e_{0}, e_{1}, e_{2}, e_{3}\right\}$ is chosen at each space-time point. This is the reason why anholonomic components come into play. One can expand the tetrads with respect to the tangent vectors along coordinate lines ('holonomic basis') according to

$$
e_{n}=e_{n}^{\mu} \partial_{\mu} .
$$

Usually one chooses the tetrad to be orthonormal,

$$
e_{n} \cdot e_{m} \equiv g_{\mu \nu} e_{n}^{\mu} e_{m}^{\nu}=\eta_{n m} \equiv \operatorname{diag}(-1,1,1,1) .
$$


The reason why one has to go beyond the pure metric formalism is the fact that spinors (describing fermions) are objects whose wave components transform with respect to a two-valued representation of the Lorentz group. One therefore needs a local Lorentz group and local orthonormal frames.

One can define anholonomic Dirac matrices according to

$$
\gamma^{n} \equiv e_{\mu}^{n} \gamma^{\mu}
$$

where $e_{n}^{\mu} e_{\mu}^{m}=\delta_{n}^{m}$. This leads to

$$
\left[\gamma^{n}, \gamma^{m}\right]_{+}=2 \eta^{n m} \text {. }
$$

The Dirac equation in curved space-time or accelerated frames then reads

$$
\left(\mathrm{i} \gamma^{n} D_{n}+\frac{m c}{\hbar}\right) \psi(x)=0
$$

In order to study quantum effects of fermions in the gravitational field of the Earth, one specializes this equation to the non-inertial frame of an accelerated and rotating observer, with acceleration a and angular velocity $\boldsymbol{\omega}$, respectively (see e.g. [24]). A non-relativistic approximation with relativistic corrections is then obtained by the standard Foldy-Wouthuysen transformation. This leads to (writing $\beta \equiv \gamma^{0}$ )

$$
\mathrm{i} \hbar \frac{\partial \psi}{\partial t}=H_{\mathrm{FW}} \psi
$$

with

$$
\begin{aligned}
H_{\mathrm{FW}}= & \beta m c^{2}-\frac{\beta \hbar^{2}}{2 m} \Delta+\beta m(\mathbf{a x})-\frac{\beta \hbar^{4}}{8 m^{3} c^{2}} \Delta^{2} \\
& -\boldsymbol{\omega}(\mathbf{L}+\mathbf{S})-\frac{\beta \hbar^{2}}{2 m} \nabla \frac{\mathbf{a} \mathbf{x}}{c^{2}} \nabla-\frac{\mathrm{i} \beta \hbar^{2}}{4 m c^{2}} \boldsymbol{\sigma}(\mathbf{a} \times \nabla)+\mathcal{O}\left(\frac{1}{c^{3}}\right)
\end{aligned}
$$

( $\sigma$ denotes the Pauli matrices). The interpretation of the various terms in (28) is straightforward. The first, second, and fourth terms correspond to the rest energy, the usual non-relativistic kinetic term, and the first relativistic correction to the kinetic term, respectively, cf. the analogous terms in the effective Hamiltonian 177 arising from the Klein-Gordon equation. The third term gives an interaction with the acceleration that is relevant for interference experiments in the gravitational field or for accelerated systems, see Sect. 2.3. (It corresponds to $-m U$ in (17).) The term $\boldsymbol{\omega} \mathbf{L}$ describes the Sagnac effect, while $\boldsymbol{\omega} \mathbf{S}$ corresponds to a spin-rotation effect ('Mashhoon effect') that cannot be found from the Schrödinger equation (but it can be found from the Pauli equation, which arises from the Dirac equation in the non-relativistic limit). One can estimate that for typical values of a neutron interferometer experiment, the Mashhoon effect contributes only $10^{-9}$ of the Sagnac effect. This is very small, but it has been indirectly observed [25]. The term after these rotation terms corresponds to the term containing $\nabla U \cdot \nabla$ in (17). (The term $\propto U^{2}$ in (17) has no analogue here because it arose from the post-Newtonian order $\propto U^{2}$ in 15, which is not considered here.)

The case of a constant gravitational acceleration $\mathrm{g}$ is obtained from (28) by replacing a with $\mathrm{g}$. The effect of curvature has thus not yet been taken into account. The Dirac equation in an external gravitational field in the post-Newtonian approximation was discussed in [26]. There, an additional external electromagnetic field has been considered, although not in a form that can be used directly for the discussion below.

If torsion were present in spacetime, it would give additional terms in 28. From an analysis of existing experiments dealing with atomic spectra, one can get stringent limit on torsion; more precisely, on the spatial component of the axial torsion, $K$, because only this component enters in this limit. The result is $K \leq 1.5 \times 10^{-15} \mathrm{~m}^{-1}$ [27]. Torsion, even if present on the fundamental level, can thus safely be neglected on the scale of laboratory physics (provided, of course, that this experimental limit holds for all components of the torsion). The Foldy-Wouthuysen transformation for an electron in an external electromagnetic field and in Minkowski space, but with non-vanishing torsion, is described in [28]. 


\subsection{Observed effects and theoretical ideas}

Observations of the interaction between quantum effects and gravity have mainly been achieved-apart from free-fall situations, for example, of freely falling Bose-Einstein condensates [29] -in the realm of neutron and atom interferometry. Colella, Overhauser, and Werner ('COW') have measured in 1975 a phase shift in a neutron's wave function caused by gravity [30], while Bonse and Wroblewski have performed in 1983 the analogous experiment for neutrons in an accelerated frame [31]. The observed effect can immediately be derived from the third term in (28). The COW experiment has in particular confirmed the validity of the weak equivalence principle for this quantum system. The quantum Sagnac effect described by the term $\omega \mathbf{L}$ in $(28$ ) has also been seen [32], as well as the Mashhoon effect. We are not aware of experiments exploiting the other correction terms in (28).

Another application of neutron interferometry is the demonstration that neutrons have a discrete energy spectrum in the gravitational field of the Earth, as predicted by the Schrödinger equation [33]. The minimum energy is $1.4 \times 10^{-12} \mathrm{eV}$, which is much smaller than the ground-state energy of the hydrogen atom. Other experiments use atom interferometry, since atoms are easier to handle and allow higher precisions in the experiments, cf. [34].

We also mention that among the many applications of future experiments in space one also attempts to study the influence of gravity on quantum entanglement [6]. An investigation of the possible lack of an influence of gravity on the quantum Hall effect can be found in [35].

Finally, we note some further ideas which have led to proposed candidates for a measurable interaction between quantum mechanics and GR: (i) the interaction of Rydberg atoms with gravitational fields [36], (ii) an atomic analogue of the LIGO interference experiment labeled the 'Matter-wave Interferometric Gravitational-wave Observatory' (MIGO), in which quantum interference between atoms replaces the classical interference between light rays [37] (this proposition has been criticized recently in [38]), and (iii) the identification of the dark energy in the universe with the quantum fluctuations as determined in noise measurements in Josephson junctions [39].

\section{Gravitational waves}

\subsection{Classical discussion}

GR has-like electrodynamics — solutions describing waves propagating with the speed of light ('gravitational waves'). While there exist exact solutions of Einstein's equations describing such waves, it is sufficient for many purposes to describe gravitational waves in the weak-field approximation, in which the waves propagate on a given background spacetime. The reason is that one expects from an observational point of view only weak gravitational waves. Starting point is thus the following decomposition of the metric, ${ }^{3}$

$$
g_{\mu \nu}=\eta_{\mu \nu}+f_{\mu \nu}
$$

in which one assumes that there exist coordinates such that the components of $f_{\mu \nu}$ are small. Introducing the combination

$$
\bar{f}_{\mu \nu} \equiv f_{\mu \nu}-\frac{1}{2} \eta_{\mu \nu} f_{\rho}^{\rho}
$$

and imposing the gauge condition $\partial_{\nu} \bar{f}_{\mu}^{\nu}=0$, one finds the linearized Einstein equations,

$$
\square \bar{f}_{\mu \nu}=-\frac{16 \pi G}{c^{4}} T_{\mu \nu}
$$

\footnotetext{
3 This formalism can be found in all standard texts on GR, see, for example, [40] or [41].
} 
The linearized level can be used both for the description of solar-system effects such as light deflection near the Sun and the description of rapidly varying fields such as gravitational waves (the situation discussed here). In the vacuum case $\left(T_{\mu \nu}=0\right)$ one finds plane-wave solutions describing waves that are transversal to the direction of propagation and have only two independent degrees of freedom (like electromagnetic waves). The two independent linear polarization states are inclined towards each other by 45 degrees and are usually referred to as the + and the $\times$ polarization. The wave thus possesses helicity two, which in quantum theory leads to the spin-2 nature of the massless graviton.

Although energy in general relativity has no local significance, one can (independent of any approximation) prove that a gravitational wave carries away positive energy to regions far away from any radiating source (the amount of energy that can be radiated away is limited by the ADM energy, which is nonnegative), see, for example, Sect. 11.2 in [42]. In the linear approximation studied here, one can find from (31) the physically relevant solution given by the retarded potential,

$$
\bar{f}_{\mu \nu}(\mathbf{x}, t)=\frac{4 G}{c^{4}} \int \mathrm{d}^{3} x^{\prime} \frac{T_{\mu \nu}\left(t-\frac{\left|\mathbf{x}-\mathbf{x}^{\prime}\right|}{c}, \mathbf{x}^{\prime}\right)}{\left|\mathbf{x}-\mathbf{x}^{\prime}\right|} .
$$

From this solution one finds in the quadrupole approximation the following 'quadrupole formula', which was already derived by Einstein in 1918, and which gives the emitted power $P$ as [40 41]

$$
P \equiv-\frac{\mathrm{d} E}{\mathrm{~d} t}=\frac{G}{5 c^{5}} \sum_{i, j=1}^{3} \frac{\mathrm{d}^{3} Q_{i j}}{\mathrm{~d} t^{3}} \frac{\mathrm{d}^{3} Q_{i j}}{\mathrm{~d} t^{3}}
$$

where

$$
Q_{i j}=\int \mathrm{d}^{3} x\left(x_{i} x_{j}-\frac{1}{3} \delta_{i j} r^{2}\right) \rho_{\mathrm{m}}
$$

denotes the mass quadrupole tensor (evaluated at retarded time). In the special case of a test mass $m$ that is freely falling on a circular orbit in the Schwarzschild metric of a mass $M \gg m$, one finds from [33,

$$
P=\frac{c^{5}}{5 G}\left(\frac{2 G M}{r c^{2}}\right)^{3}\left(\frac{2 G m}{r c^{2}}\right)^{2} .
$$

The prefactor has the dimension of power, $c^{5} / 5 G \approx 7.29 \times 10^{51}$ Watt, but this would only be released if $m$ could fall close to the Schwarzschild radius corresponding to $M$. For the Earth moving around the Sun, the power emitted in gravitational waves is only about 200 Watt.

In laboratory situations the emission of gravitational waves is usually estimated to be utterly negligible. Considering, for example, a rod of mass $M$ and length $L$ spinning about its centre with frequency $\omega$, one finds from (33) for the emitted power,

$$
P=\frac{2 G}{45 c^{5}} M^{2} L^{4} \omega^{6},
$$

which upon inserting typical values such as $M=1 \mathrm{~kg}, L=1 \mathrm{~m}, \omega=1 \mathrm{~Hz}$ yields the utterly small value $P \approx 10^{-54}$ Watt. One would thus need a very strong gravitational field (such as the one present in the coalescence of two compact objects) to get a measurable effect. This has to be kept in mind when discussing proposals aiming at generating sufficiently strong gravitational waves in the laboratory through quantum effects (Sect. 3.2).

Gravitational waves have hitherto only been observed indirectly through the decrease of the orbital period of the binary pulsar PSR 1913+16 (and other pulsars as well). Attempts to detect (not generate) gravitational waves on Earth are presently underway with the help of laser interferometers, some of which have started to operate in 2002, cf. [43]. These interferometers should be able to detect waves with an 
amplitude $h$, describing the relative change in separation of two test masses, of $h \sim 10^{-21}$ (for short signals) and down to $h \sim 10^{-26}$ (for a signal of a duration of one year). They have a frequency window from about $10 \mathrm{~Hz}$ to $10 \mathrm{kHz}$. The envisaged space mission LISA (to be launched in 2012) will be able to measure frequencies down to about $10^{-4} \mathrm{~Hz}$.

In addition to theses interferometers, there are also resonance cylinders ('Weber bars') in use. The first detectors of this kind have been developed already in the sixties. They have the disadvantage of being sensitive only around a specific (resonant) frequency and to relatively large amplitudes. It was estimated that the efficiency of emitting gravitational radiation by such a cylinder is [44]

$$
\eta_{\mathrm{rad}}=\frac{\Gamma_{\text {grav }}}{\Gamma_{\text {heat }}}=\frac{64 G M v_{\mathrm{s}}^{4}}{15 L^{2} c^{5} \Gamma_{\text {heat }}} \approx 10^{-34},
$$

where $M$ is the mass of the bar, $L$ its length, $\Gamma_{\text {heat }}$ is the decay rate due to dissipation, and $v_{\mathrm{s}}$ is the speed of sound. In the classical (Weber bar) case, we have $v_{\mathrm{s}} \approx 10^{-5} \mathrm{c}$. The emission of gravitational waves by a Weber bar is thus utterly negligible. Suggestions about how this may change by a coupling to quantum systems will be discussed in the following subsection.

\subsection{Generation and detection of gravitational waves via quantum systems}

In the last section, the concepts of classical gravitational waves as solutions to the linearized Einstein equations were introduced. Despite the ongoing experiments to detect gravitational waves, the search has been unsuccessful so far, although the interferometers mentioned above are expected to lead to a detection in the future. In addition, there have been some approaches which suggest that the interaction of gravitational waves with quantum, instead of classical matter should be enhanced and would thus lead to better detection results. These ideas rest on certain properties of quantum fluids - for example, superconductors, superfluids, quantum Hall (QH) fluids, and atomic Bose-Einstein condensates (BECs) - exhibiting macroscopic coherence which is absent in classical materials due to the strong entanglement with the environment which leads to decoherence [7].

The general interaction of gravitational fields with quantum fluids has been extensively studied. Already DeWitt [45] and Papini [46, 47, 48] investigated the interaction of Thirring-Lense fields with rotating superconductors and considered the induced quantum phase shift (see also Sect. 4). In [49], Anandan and Chiao considered constructing antennas for gravitational radiation using superfluids, while [50] considers the detection of gravitational radiation with superconducting circuits. In [51], Peng and Torr investigated the coupling of gravitational waves to superconductors, while Speliotopoulos found a cross-coupling term between the electromagnetic and gravitational field in the long-wavelength approximation, which could lead to a conversion between the two fields [52]. Tajmar and de Matos considered a classical coupling between electromagnetic and gravitoelectromagnetic waves ${ }^{4}$ [53, 54], which, however, would lead to a very small interaction strength (cf. Sect. 4). Chiao et al. [55, 56 57] considered using quantum fluids as transducers, that is, as generator as well as detector of gravitational waves. Finally, the idea of conversion between gravitational and electromagnetic waves was recently discussed in [58].

We focus here on the ideas which describe the generation (and detection) of gravitational waves via the use of quantum fluids. First, the concept of a quantum fluid is discussed and its different properties in comparison to classical matter are addressed. Then, we investigate the arguments used in the research to suggest that quantum fluids should be better interaction partners of gravitational waves than classical materials. We consider the proposed coupling schemes and discuss their legitimacy. Finally, we discuss another investigation of the conversion between electromagnetic and gravitational waves as well as the experiments which have been conducted so far.

\footnotetext{
${ }^{4}$ Such a coupling eventually has to be derived from the coupled Einstein-Maxwell equations.
} 


\subsubsection{Classical materials versus quantum fluids}

The important property of quantum fluids which is central to the interaction schemes proposed above is the existence of a macroscopic quantum-mechanical phase coherence which is not suppressed by interaction with the environment, such as, for example, phonons or photons in conductors and semiconductors. Macroscopic coherence is generated as a result of the condensation processes in the systems; this coherence is absent in classical materials.

In superconductors, the coherent ground state consists of a condensate in the form of Cooper pairs, separated from the excited states by an energy gap, the so-called BCS gap, see, for example, [59]. In the quantum Hall fluid, which is realized by a quasi-two dimensional electron gas confined by a strong magnetic field, the electrons form composite fermions with an even number of flux quanta to form a $\mathrm{QH}$ condensate at low temperatures. The ground state is energetically protected from decoherence by the $\mathrm{QH}$ gap [60, 61]. Superfluids and atomic BECs also show macroscopic phase coherence in the formation of condensates at low temperatures, see, for example, [62]. As long as the transition to excited states is negligible, the coherences are not suppressed, and thus the reduced density matrix shows non-vanishing off-diagonal terms over spatially large distances (for this reason, one refers to these materials as quantum many-body systems with off-diagonal long-range order (ODLRO) [63]).

It is the existence of these coherences which is argued to lead to a stronger interaction with gravitational fields and a more efficient generation (and detection) of electromagnetic and gravitational waves.

3.2.2 General remarks about the (classical) interaction strength of gravitational waves with quantum fluids

In [55, 56], it is argued heuristically that gravitational waves should couple more strongly to quantum fluids than to classical materials because, due to the macroscopic phase coherence, the length scale of the material is (in principle) not limited to an acoustic wavelength $\lambda_{\mathrm{s}}=v_{\mathrm{s}} T$ of the material, where $v_{\mathrm{s}}$ is again the speed of sound and $T$ the period of the gravitational wave. Due to the classical coupling via acoustic waves, classical matter used as an antenna for gravitational waves is restricted to roughly the size $\lambda_{\mathrm{s}}$. In the quantum coupling, this restriction may not apply. Since the macroscopic quantum coherences in quantum fluids can in principle be of the same length scale as the gravitational wavelength $\lambda$, it is suggested that one could in principle, because the length restrictions do not apply in quantum fluids, replace the speed of sound $v_{\mathrm{s}}$ in the material by the speed of light $c$.

From Sect. 3.1 we know that for the efficiency of emitting gravitational radiation by a cylindrical bar, $\eta_{\mathrm{rad}} \sim v_{\mathrm{s}}^{4}$ (see (37). Taking an approximate speed of sound in classical materials of $v_{\mathrm{s}} \approx 10^{-5} c$, Chiao's argument suggests an increased radiation emission efficiency of $\left(c / v_{\mathrm{s}}\right)^{4} \approx 10^{20}$. It should be noted here that, in contrast to the classical considerations of gravitational-wave detection, where (astrophysical) frequencies in the range of $\mathrm{Hz}$ to $\mathrm{kHz}$ are considered, the frequencies, corresponding to the above arguments of wavelengths on the same length scale as the detector, would be of the order of $10 \mathrm{MHz}$ and higher (see Sect. 3.2.4 for an experiment conducted to this end). No detectable astrophysical sources are available in this frequency range.

Furthermore, it is suggested that through 'impedance' matching of the gravitational wave to the quantum fluid, an enhanced conversion between the two fields can be obtained. In the electromagnetic case, the impedance of free space is given by

$$
Z_{0}=\sqrt{\frac{\mu_{0}}{\epsilon_{0}}} \approx 377 \Omega
$$


where $\epsilon_{0}$ and $\mu_{0}$ are the electric permittivity and magnetic permeability, respectively. Analogously, the 'impedance' for the gravitational wave is given by ${ }^{5}$

$$
Z_{\mathrm{g}}=\sqrt{\frac{\mu_{\mathrm{g}}}{\epsilon_{\mathrm{g}}}}=\frac{4 \pi G}{c} \approx 2.79 \times 10^{-18} \mathrm{~m}^{2} / \mathrm{kg} \mathrm{s},
$$

where $\epsilon_{\mathrm{g}}$ and $\mu_{\mathrm{g}}$ are the gravitational permittivity and permeability of free space, respectively. Considering an electromagnetic wave impinging normally on a thin resistive film, the absorption, transmission, and reflection coefficients depend on the relationship between the resistance of the material and the impedance of free space $Z_{0}[64 \mid$. It is now argued that, solving a similar boundary problem for the gravitational wave, an analogous 'impedance' matching condition can be derived. While in classical materials, the 'resistance' is generally much higher than $Z_{\mathrm{g}}$, leading to virtual transparency with respect to the gravitational wave, quantum materials such as superconductors could have a 'resistance' comparable to that of $Z_{\mathrm{g}}$ which could lead to maximal absorption of the gravitational wave. If the absorption could be viewed as an effective dissipation where gravitational-wave energy is converted into electromagnetic-wave energy instead of into heat, this would imply a reduced dissipation $\Gamma_{\text {heat }}$, leading to an enhanced conversion process [56]. These expectations are, however, based on heuristic considerations and not on detailed calculations.

From the above arguments, one could expect that couplings between gravitational fields and quantum fluids be enhanced compared to the coupling to classical matter. However, even with the increase of the efficiency, as argued by Chiao, it is questionable whether this leads to effects which are large enough to be detected. Furthermore, it is important to note that the arguments are based on the classical efficiency formula derived by Weinberg [44]. Since this derivation is based on classical properties of the detector such as sound waves in the material, it is not clear whether these arguments can be applied to quantum fluids. Generally, a theory regarding the quantum nature of the (macroscopic) coherences would have to be considered. It should be noted that (classical) Weber bars have been studied in the quantum regime by using quantum nondemolition measurements (see e.g. [65 66]). A quantum measurement analysis is also crucial for the detection of gravitational-wave signals in current interferometer experiments [67].

\subsubsection{Quantum-mechanical coupling considerations}

Now we want to discuss the quantum-mechanical arguments used to explain the coupling between the electromagnetic and gravitational fields. Again, these are based on the existence of macroscopic phase coherence that leads to the generation of electromagnetic radiation and, by means of time reversal, to the detection of electromagnetic waves by generating gravitational waves [55].

It is well known that, in the presence of an electromagnetic potential $A_{\mu}$, the quantum-mechanical wave function of a charged particle picks up a phase (the Aharonov-Bohm phase [68]),

$$
\Psi(\mathbf{x}) \rightarrow \Psi(\mathbf{x}) \exp \left(\frac{\mathrm{i} e}{\hbar c} \int A_{\mu} \mathrm{d} x^{\mu}\right),
$$

which has been experimentally detected by means of interference experiments, see, for example, [69]. More generally, if a system evolves adiabatically (i.e., if the quantum adiabatic theorem holds) in a parameter space $\{\mathbf{R}\}$ around a closed curve $C$ in the presence of a non-trivial topology, the wave function accumulates a (topological) Berry phase [70] 71],

$$
\Psi_{n}(\mathbf{x}) \rightarrow e^{\mathrm{i} \gamma_{n}(C)} \Psi_{n}(\mathbf{x}),
$$

\footnotetext{
${ }^{5}$ It is shown in Sect. 4.1.1 that the linearized Einstein equations can be written in a form analogous to Maxwell's equations, thus allowing for the definition of gravitational analogues of the electric permittivity and magnetic permeability. In the definition of the 'impedance' for the gravitational wave, we have used SI units in order to show the analogy to the electromagnetic case. Note that in comparison to [56, the gravitational 'impedance' $Z_{\mathrm{g}}$ defined above is smaller by a factor 4, which is due to the different definitions of the gravitoelectromagnetic potentials, see Sect. 4.1.1
} 
where the phase is explicitly given by

$$
\gamma_{n}(C)=\mathrm{i} \oint_{C}\left\langle n(\mathbf{R}) \mid \nabla_{\mathbf{R}} n(\mathbf{R})\right\rangle \cdot \mathrm{d} \mathbf{R},
$$

where $|n(\mathbf{R})\rangle$ are eigenstates of the system. The Berry phase has been experimentally detected, for example, by considering the polarization of neutrons in the presence of a spiral magnetic field [72] (see also [73, 74]).

In the Aharonov-Bohm case, a time-dependent phase leads to an 'electromotive' force which acts on the electrons. Similarly, a Berry phase can, in the case of a coupling of the spin with an external field, lead to a '(nonelectro)motive' force which acts on the spin of the particle. In both cases, this is a result of the fact that the free energy of the system is dependent on the quantum-mechanical phase; the result is an electric, non-dissipative equilibrium (persistent) current in the mesoscopic system [75] (for an experimental measurement of the Aharonov-Bohm persistent current, see e.g. [76]).

It has been shown by DeWitt [45] and Papini [47] that in the case of weak gravitational fields and slow velocities (not suitable to describe gravitational waves), the so-called gravitomagnetic potential $\mathbf{A}_{\mathrm{g}}$ (cf. Sect. 4.1.1, arising from the gravitational field, leads to a Berry-type phase

$$
\Delta S \sim \oint \mathbf{A}_{\mathrm{g}} \cdot \mathrm{d} \mathbf{l} .
$$

It is now argued by Chiao et al. [55] that in the presence of a gravitational wave, the electron wave function should also pick up a Berry-type phase which, due to its spin, would lead to a macroscopic electric current which, in turn, would produce electromagnetic waves. Assuming time-reversability, the opposite process of the conversion of electromagnetic waves into gravitational waves should also be possible.

Now let us turn to the mathematical model involved in the coupling. The different models which are discussed in the literature consider the case of minimally coupled electromagnetic and gravitational fields. The classical derivations, considered, for example, by DeWitt [45] and elaborated by Papini [46] 47], start from the Lagrangian of a 'classical' electron in an electromagnetic and weak gravitational field (see Sect. 4.2.1 for details), without considering gravitational waves. This leads to a Hamiltonian with cross-coupling terms of the form

$$
H_{\text {int }} \sim \mathbf{A} \cdot \mathbf{A}_{\mathrm{g}},
$$

where $\mathbf{A}$ and $\mathbf{A}_{\mathrm{g}}$ are the magnetic and gravitomagnetic 3-potentials. Speliotopoulos also obtained terms of the above form in his long-wavelength approximation model [52].

Coupling of the electron including its quantum-mechanical properties via minimal coupling in the Dirac equation as in Sect. 2.2. but including here the electromagnetic field as well, is achieved by (cf. Sect. 2.2 for details on the index notation)

$$
p_{\mu} \rightarrow p_{\mu}-\frac{e}{c} A_{\mu}+\frac{\hbar}{2} \sigma^{m k} \Gamma_{\mu m k} .
$$

For the purely gravitational case, the non-relativistic expansion of this Dirac equation coincides with the prescription of [22]. As far as we know, there has not been a general systematic derivation of the nonrelativistic Hamiltonian in the presence of both electromagnetic and gravitational fields, neither in a loworder post-Newtonian approximation such as is used in the derivation of 28, nor in the background of a gravitational wave. The results of [26] employ a post-Newtonian approximation (where gravitational waves are not seen) with an application to the hydrogen atom. It is thus questionable whether the nonrelativistic result will contain, as proposed by Chiao et al. [55], in the effective Hamiltonian a term of the form

$$
H=\frac{1}{2 m}\left(p_{i}-\frac{e}{c} A_{i}+G_{i}\right)^{2}+V
$$


where $G_{i}$ denotes the non-relativistic form emerging from the minimal coupling to the gravitational field in (45). On algebraic grounds, it could certainly be possible that the resulting coupling terms between the electromagnetic and gravitational fields vanish due to the properties of the $\gamma$-matrices. It is, however, highly recommended that this derivation be performed in order to gain insight into the possibility of such a coupling as well as its strength.

If one assumes the above form for the interaction Hamiltonian, one is led directly to cross-coupling terms

$$
H_{\mathrm{int}} \sim \mathbf{A} \cdot \mathbf{G}
$$

where $\mathbf{A}$ is the magnetic 3-potential and $\mathbf{G}$ incorporates the gravitational field; in comparison to the results derived by DeWitt and Papini, these terms would include the quantum-mechanical coupling between the gravitational and electromagnetic field via the spin of the electron. It should be noted here that in a conversion process between electromagnetic and gravitational fields described by (47), momentum conservation must be obeyed, either by the addition of momentum as supplied by the medium (e.g. strong magnetic fields in the quantum Hall fluid) or by considering electromagnetic quadrupole fields (e.g. in the conversion processes involving superconductors) [57].

\subsubsection{Experimental and quantitative theoretical results}

We know of only one experiment which has been carried out in a laboratory framework where a quantum fluid is supposed to act as a transducer between gravitational and electromagnetic waves. In this experiment [57], superconducting yttrium barium copper oxide (YBCO) was used as a transducer. Two probes were used, one as a generator and the other as a detector of gravitational waves. Electromagnetic quadrupole radiation with a frequency of $12 \mathrm{GHz}$ is shone upon the first transducer, producing gravitational radiation which falls upon the second superconductor, where the generated electromagnetic quadrupole radiation is detected. In order to prevent direct electromagnetic coupling, the two superconductors are enclosed by Faraday cages.

At least in this relatively simple setup, there has been no detection of generated gravitational waves, which, according to Chiao et al. [57], gives an upper conversion efficiency of $\eta<1.6 \times 10^{-5}$ (if one assumes equal conversion efficiencies from one form of wave to another). Chiao et al. argue that this negative result may be due to

- possible high residual microwave and far-infrared losses in $\mathrm{YBCO}$, or

- generally large ohmic dissipation arising from high temperatures; this could be reduced at lower temperatures.

It is possible that the experimental conditions could be improved for this situation. However, since there is as yet no evidence for a coupling of such strength as to be detectable, it would be preferable first to investigate that matter theoretically.

Finally, we want to shortly comment on calculations performed by Licht [58]. He considers gravitational waves impinging on normal conductors as well as superconductors and calculates, using classical electrodynamical arguments, the generation efficiency of outwardly propagating electromagnetic waves. The superconductor is described within a phenomenological Ginzburg-Landau approach (see Sect. 4.1.3). The result is that the generation of electromagnetic waves in superconductors in both the low and high frequency domain is not strongly enhanced compared to that in normal conductors and should not lead to detectable results. It should be noted, however, that in comparison to the argumentation of Chiao et al., no quantum-mechanical properties such as the spin of the electron have been considered in these calculations. 
3.2.5 Conclusion on the attempts to generate and detect electromagnetic waves in quantum systems

To summarize the proposed ideas of using quantum fluids as transducers between electromagnetic and gravitational waves:

There have been arguments presented for the case of a stronger coupling of gravitational waves with quantum fluids than is the case with classical materials. Heuristic considerations of Weinberg's formula (37) for the emission efficiency of gravitational waves from a (classical) Weber bar have been presented to give enhancements for quantum fluids. Yet, it is not clear as to whether these arguments can be applied to this formula since its derivation is based on classical properties. As far as the arguments based on the Dirac equation are concerned, to our knowledge there is no non-relativistic derivation available which includes both electromagnetic and gravitational field in a general post-Newtonian approximation (which in low order does not describe gravitational waves) or in the background of a gravitational wave. In order to make qualitative as well as quantitative comments on the coupling strength, it is highly recommended to perform this derivation.

The theoretical investigations performed by Licht [58] lead to generation efficiencies which are so small as not to be relevant for experimental laboratory research. Since the experiments conducted by Chiao et al. have also not yet led to positive results, it would be advisable that the interaction first be further explored on a theoretical basis before new experiments are carried out.

\section{Gravitational effects in superconductors}

In the last section, we have discussed proposed interaction mechanisms and experiments between gravitational and electromagnetic fields, where the emphasis was on the use of quantum systems as detector and generator of gravitational waves. Here, we will discuss interaction processes involving weak gravitational fields coupled to rotating superconductors, where the emphasis is on small velocities, thus specifically excluding gravitational waves. We will review the work which has been done in this area so far and the different mechanisms which have been proposed and give a critical discussion of the ideas and the possible realizations and measurements of such interactions.

\subsection{Foundations}

To begin, it is necessary to present the basic concepts which are used. First, we introduce the concept of gravitoelectromagnetism, which follows in the weak-field approximation of GR for slowly moving matter. This is a valid approximation as long as we are concerned with laboratory experiments which do not deal with gravitational waves. We will then mention a proposed classical coupling scheme between electro- and gravitoelectromagnetism which has been discussed in the literature. Finally, we introduce the concepts for superconductors which are important for the argumentations used in the current approaches.

\subsubsection{Gravitoelectromagnetism}

Within the linear approximation of GR, gravitational analogues of the electric and magnetic fields of Maxwell's theory of electromagnetism can be derived if we assume, in addition, that we are only considering slowly moving objects [77, 78, 79]. The field equations can be formulated in a Maxwell-type structure. In this section, we will mention the most important concepts of this so-called theory of gravitoelectromagnetism, using the notation of [79]. ${ }^{6}$

\footnotetext{
6 There is a certain degree of arbitrariness in the definition of the gravitoelectromagnetic potentials. The notation used here is chosen as to clearly show the analogy to the electromagnetic case, while also pointing out the difference (as witnessed in the factor $1 / 2$ for the gravitomagnetic field which stems from the ratio of gravitomagnetic to gravitoelectric charge $q_{\mathrm{B}} / q_{\mathrm{E}}=2$ ).
} 
The following derivation is similar to that for gravitational waves (see Sect. 3.1]. It is assumed that only weak perturbations of flat spacetime occur, so that we may again consider $g_{\mu \nu}=\eta_{\mu \nu}+f_{\mu \nu}$, where $f_{\mu \nu}$ is small. The Einstein equations then reduce to (31). However, in contrast to the derivation of gravitational waves, we take here as a source an ideal fluid, assuming velocities small compared to $c$ : $T^{00}=\rho_{\mathrm{m}} c^{2}$, $T^{0 i}=c j^{i}$, and $T^{i j}=\mathcal{O}\left(c^{0}\right)$, where $\rho_{\mathrm{m}}$ is the matter density and $\mathbf{j}=\rho_{\mathrm{m}} \mathbf{v}$ is the matter current density.

If we now introduce the (scalar) gravitoelectric and (vector) gravitomagnetic potentials,

$$
\Phi_{\mathrm{g}}:=\frac{c^{2}}{4} \bar{f}_{00}, \quad\left(A_{\mathrm{g}}\right)_{i}:=-\frac{c^{2}}{2} \bar{f}_{0 i}
$$

respectively, we can write the Einstein equations up to $\mathcal{O}\left(c^{-4}\right)$ in the following form:

$$
\begin{array}{ll}
\nabla \cdot \mathbf{E}_{\mathrm{g}}=4 \pi G \rho_{\mathrm{m}} & \nabla \times\left(\frac{1}{2} \mathbf{B}_{\mathrm{g}}\right)=\frac{1}{c} \partial_{t} \mathbf{E}_{\mathrm{g}}+\frac{4 \pi G}{c} \mathbf{j} \\
\nabla \times \mathbf{E}_{\mathrm{g}}=-\frac{1}{c} \partial_{t}\left(\frac{1}{2} \mathbf{B}_{\mathrm{g}}\right) & \nabla \cdot\left(\frac{1}{2} \mathbf{B}_{\mathrm{g}}\right)=0 .
\end{array}
$$

Here, we have introduced the gravitoelectric and gravitomagnetic fields

$$
\begin{aligned}
& \mathbf{E}_{\mathrm{g}}=-\nabla \Phi_{\mathrm{g}}-\frac{1}{c} \partial_{t}\left(\frac{1}{2} \mathbf{A}_{\mathrm{g}}\right), \\
& \mathbf{B}_{\mathrm{g}}=\nabla \times \mathbf{A}_{\mathrm{g}},
\end{aligned}
$$

respectively.

It should be noted that the gravitoelectric and gravitomagnetic potentials defined above are just the Newtonian potential and 'Thirring-Lense potential', respectively. This can be seen by regarding the solutions to 31 , given by (32),

$$
\bar{f}_{\mu \nu}(\mathbf{x}, t)=\frac{4 G}{c^{4}} \int \mathrm{d}^{3} x^{\prime} \frac{T_{\mu \nu}\left(t-\frac{\left|\mathbf{x}-\mathbf{x}^{\prime}\right|}{c}, \mathbf{x}^{\prime}\right)}{\left|\mathbf{x}-\mathbf{x}^{\prime}\right|} .
$$

If one considers a source distribution confined around the spatial coordinate origin, the potentials far away from the source can be written as

$$
\Phi_{\mathrm{g}} \sim \frac{G M}{r}, \quad \mathbf{A}_{\mathrm{g}} \sim \frac{G}{c} \frac{\mathbf{J} \times \mathbf{x}}{r^{3}},
$$

where $r=|\mathbf{x}|$, and $M$ and $\mathbf{J}$ are the mass and angular momentum of the source, respectively.

In order to point out the difference between linearized GR and electromagnetism, we remark that within the above approximations, it is possible to derive a Lorentz-type force in the lowest order of $\mathbf{v} / c$ and assuming $\mathbf{A}_{\mathrm{g}}$ is time-independent, yielding

$$
\mathbf{F}=-m \mathbf{E}_{\mathrm{g}}-2 m \frac{\mathbf{v}}{c} \times \mathbf{B}_{\mathrm{g}}
$$

Introducing, in analogy to electromagnetism, the gravitoelectric and gravitomagnetic charges $q_{\mathrm{E}}=-m$ and $q_{\mathrm{B}}=-2 m$, respectively, we have the ratio $q_{\mathrm{B}} / q_{\mathrm{E}}=2$, compared to $q_{\mathrm{B}} / q_{\mathrm{E}}=1$ in the electromagnetic case. This can be traced back to the fact that linearized gravity is a spin-2 theory, whereas electromagnetism is fundamentally spin- 1 . For a rotating body of mass $M$ that acts as a source, the gravitational charges are given by $q_{\mathrm{E}}=M$ and $q_{\mathrm{B}}=2 M$ to preserve the attractive nature of GR. ${ }^{7}$

\footnotetext{
7 Here, the Gaussian system of units has been used; however, it is helpful to bring the gravitoelectromagnetic field equations into SI form in order to introduce the concepts of the gravitoelectric permittivity and gravitomagnetic permeability. In order to define the gravitoelectromagnetic fields in an analogous way as in electromagnetism,
} 


\subsubsection{Classical coupling between gravitoelectromagnetism and electromagnetism}

Based on the above analogy, a coupling between the electromagnetic and gravitoelectromagnetic fields can be derived [53 80]. The matter density $\rho_{\mathrm{m}}$ and charge density $\rho$ of a point-like or uniformly distributed mass and charge configuration of mass $m$ and charge $e$ obey

$$
\rho_{\mathrm{m}}=\frac{m}{e} \rho .
$$

From this, the following relationships between the potentials can be derived,

$$
\begin{aligned}
& \Phi_{\mathrm{g}}=G \frac{m}{e} \Phi \equiv \kappa_{\mathrm{E}} \Phi, \\
& \mathbf{A}_{\mathrm{g}}=G \frac{2 m}{e} \mathbf{A} \equiv \kappa_{\mathrm{B}} \mathbf{A} .
\end{aligned}
$$

Thus, a coupling can be defined as ${ }^{8}$

$$
\kappa_{\mathrm{E}(\mathrm{B})}=G \frac{q_{\mathrm{E}(\mathrm{B})}}{e} \approx 6.67 \times 10^{-8} \frac{q_{\mathrm{E}(\mathrm{B})}}{e} \mathrm{~cm}^{3} / \mathrm{g} \mathrm{s}^{2},
$$

where $q_{E(B)}$ are the gravitoelectric and gravitomagnetic charges of the source, respectively. This leads to relationships between the electromagnetic and gravitoelectromagnetic fields,

$$
\begin{aligned}
& \mathbf{E}_{\mathrm{g}}=\kappa_{\mathrm{E}} \mathbf{E}, \\
& \mathbf{B}_{\mathrm{g}}=\kappa_{\mathrm{B}} \mathbf{B} .
\end{aligned}
$$

Due to this coupling, the motion of a particle with both mass and charge should at the same time produce an electromagnetic as well as a gravitoelectromagnetic field. Also, if one combines eqs. (58) and (59) with Maxwell's equations, gravitoelectromagnetic fields should generate electromagnetic fields and vice versa.

Note, however, that even for increased values of the ratio $m / e$, for example, for moving ions, this coupling is tiny, since $\kappa_{\mathrm{E}(\mathrm{B})} \ll m / e$.

\subsubsection{Theories of superconductivity}

This section will be devoted to a short overview of the important concepts involving superconductors insofar as they will be applied in the upcoming discussions. For further information on these theories, we refer to [59 81 82]. Since the argumentations in the current experiments are based on the London, and more generally, the Ginzburg-Landau, theory, we will restrict ourselves mainly to phenomenological descriptions of superconductivity, making only a few comments on the microscopic BCS theory. Furthermore, we

$$
\mathbf{E}_{\mathrm{g}}=-\nabla \Phi_{\mathrm{g}}-\partial_{t} \mathbf{A}_{\mathrm{g}}, \quad \mathbf{B}_{\mathrm{g}}=\nabla \times \mathbf{A}_{\mathrm{g}},
$$

it is necessary to define the potentials in a different way (see [53]),

$$
\Phi_{\mathrm{g}}:=\frac{c^{2}}{4} \bar{f}_{00}, \quad\left(A_{\mathrm{g}}\right)_{i}:=-\frac{c}{4} \bar{f}_{0 i} .
$$

The SI form of the field equations then reads

$$
\begin{array}{ll}
\nabla \cdot \mathbf{E}_{\mathrm{g}}=\frac{\rho_{\mathrm{m}}}{\epsilon_{\mathrm{g}}} & \nabla \times \mathbf{B}_{\mathrm{g}}=\frac{1}{c^{2}} \partial_{t} \mathbf{E}_{\mathrm{g}}+\mu_{\mathrm{g}} \mathbf{j} \\
\nabla \times \mathbf{E}_{\mathrm{g}}=-\partial_{t} \mathbf{B}_{\mathrm{g}} & \nabla \cdot \mathbf{B}_{\mathrm{g}}=0,
\end{array}
$$

with the gravitoelectric permittivity and gravitomagnetic permeability

$$
\epsilon_{\mathrm{g}}=\frac{1}{4 \pi G} \text { and } \mu_{\mathrm{g}}=\frac{4 \pi G}{c^{2}},
$$

respectively.

${ }^{8}$ It should be noted that in 80 , the coupling constants have been defined in SI units and are given by $\kappa_{\mathrm{E}(\mathrm{B})}=4 \pi G \epsilon_{0} \frac{q_{\mathrm{E}(\mathrm{B})}}{e} \approx$ $7.41 \times 10^{-21} \frac{q_{\mathrm{E}(\mathrm{B})}}{e} \mathrm{C}^{2} / \mathrm{kg}^{2}$. 
will restrict ourselves to type I superconductors, where the Meissner effect is strictly valid up to the critical magnetic field where superconductivity is destroyed.

London set up a phenomenological theory which explains the two unique features of a superconductor, the Meissner effect and the loss of resistance below a critical temperature $T_{c}$. The input consists of Maxwell's equations together with the two 'material equations'

$$
\begin{array}{r}
\partial_{t}\left(\Lambda \mathbf{j}_{\mathrm{s}}\right)=\mathbf{E}, \\
\nabla \times\left(\Lambda \mathbf{j}_{\mathrm{s}}\right)+\frac{1}{c} \mathbf{B}=0,
\end{array}
$$

relating the current density $\mathbf{j}_{s}$ of the superconducting electrons and the electromagnetic fields in the superconductor. Here, $\Lambda=m / e^{2} n_{\mathrm{s}}$, where $n_{\mathrm{s}}, m$, and $e$ are the density, the mass, and the charge of the superconducting electrons, respectively, is related to the London penetration depth $\lambda$-determining the degree to which a magnetic field can penetrate into a superconductor — by $\lambda=\left(\Lambda c^{2} / 4 \pi\right)^{1 / 2}[59]$.

If one takes a multiply connected superconducting material and considers the flux through an area $S$ bordered by a curve $C$ around a hole, one finds (from the single-valuedness of the wave function) that

$$
\oint_{S} \mathbf{B} \cdot \mathrm{d} \mathbf{S}+c \oint_{C} \Lambda \mathbf{j}_{\mathrm{s}} \cdot \mathrm{d} \mathbf{l}=n \frac{h c}{2 e},
$$

where $n$ is an integer which depends on the initial conditions of the superconducting system. This result can also be derived by applying the Bohr-Sommerfeld quantization condition to the local mean value of the canonical momentum $\mathbf{p}_{\mathrm{s}}=m \mathbf{v}_{\mathbf{s}}+\frac{e}{c} \mathbf{A}$ of the superconducting electrons around a closed path, ${ }^{9}$

$$
\oint_{C}\left(m \mathbf{v}_{\mathrm{s}}+\frac{e}{c} \mathbf{A}\right) \cdot \mathrm{d} \mathbf{l}=n \frac{h}{2},
$$

where $\mathbf{v}_{\mathrm{s}}$ is the velocity of the superconducting electrons, related to the current density by $\mathbf{j}_{\mathrm{s}}=n_{\mathrm{s}} e \mathbf{v}_{\mathrm{s}}$.

If the above curve runs several penetration depths in the material, the current density $\mathbf{j}_{\mathrm{s}}$ due to the superconducting electrons vanishes, and one obtains magnetic flux quantization through the area $S$. If the body is simply connected, the flux vanishes (Meissner effect).

If, on the other hand, one takes a superconducting body and rotates it, a (homogeneous) magnetic field is induced along the axis of rotation. This is caused by a weak surface current as a result of the superconducting electrons lagging behind the positive rest charges of non-superconducting electrons and nuclei (see [83] for an explanation of this effect in terms of Coriolis forces). The magnetic field induced in a body rotating with angular velocity $\boldsymbol{\omega}$ can be calculated from the quantization condition (62) to be [81, 84

$$
\mathbf{B}=-\frac{2 m c}{e} \boldsymbol{\omega}
$$

deep inside the superconducting material; it is called the London moment. In this derivation, it is used that for the initial condition of vanishing external magnetic fields and the superconducting body at rest, $n=0$ occurs in the quantization condition.

More generally, the theory of superconductivity can be considered within the Ginzburg-Landau theory, which reproduces the London equations [59]. Integrating the total current density $\mathbf{j}$ around a closed loop,

\footnotetext{
9 The factor 2 on the right-hand side of 63 arises from the fact that the superconducting electrons occur in pairs (Cooper pairs) and thus carry charge $2 e$ and mass $2 m$.
} 
and including the effects of rotation, one obtains [82, 85]

$$
\oint_{S} \mathbf{B} \cdot d \mathbf{S}+\frac{m c}{e^{2} n_{\mathrm{S}}} \oint_{C} \mathbf{j} \cdot \mathrm{d} \mathbf{l}+\frac{2 m c}{e} \boldsymbol{\omega} \cdot \mathbf{S}=n \frac{h c}{2 e},
$$

where $C$ is a curve within the superconductor with associated surface $S$, and $n_{\mathrm{s}}$ is again the density of the superconducting electrons. The third term has arisen in addition to 62. It can easily be seen that for an area $S$ bordered by a curve which lies many penetration depths in the superconductor, where the current density $\mathbf{j}$ vanishes (this can be fulfilled e.g. for a thick superconducting ring), and in the case of an initially non-rotating superconductor $(n=0)$, the above equation again leads to the London moment. For a thin superconducting ring, it is found that for every $n$ there exists an angular velocity $\omega_{n}$ where the current density $\mathbf{j}$ and the magnetic flux $\oint_{S} \mathbf{B} \cdot \mathrm{d} \mathbf{S}$ vanish together [82], leading to

$$
\frac{h}{2 m}=2 S \Delta \omega
$$

where $\Delta \omega=\omega_{n+1}-\omega_{n}$; this equation may be used to determine the mass of the Cooper pairs $m^{\prime}=2 m$ [86] (see also [82] for relativistic mass corrections).

On a microscopic level, superconductivity can be explained by the theory first devised by Bardeen, Cooper, and Schrieffer ('BCS' theory) [87] (for an introduction, see [59]). Within this theory, the ground state of a superconductor is described as a condensate of Cooper pairs, pairs of electrons which are bound by an effective electron-electron interaction mediated by the electron-phonon interaction of the electrons with the lattice. The theory also derives the so-called BCS gap between the ground and excited states of the superconductor, which leads to macroscopic coherence in the system (see Sect. 3.2.1). The GinzburgLandau theory can be derived from the BCS theory, if (i) the temperature of the system is close to the critical temperature $T_{c}$ and (ii) the London penetration depth $\lambda$ is much greater than the coherence length $\xi$ of the Cooper pairs.

\subsection{Rotating Superconductors}

Now we will discuss the effects of gravitational fields on rotating superconductors in order to review the proposed effects and to assess the planned experiments and measurement possibilities.

\subsubsection{Earlier considerations}

As mentioned above, London [81] showed that if a superconducting sphere is set in rotation, a magnetic field is generated along the axis of rotation, which is a result of the momentum quantization condition. In this derivation, only the electromagnetic properties of the electrons have been used. The effect has been experimentally confirmed, see, for example, [88, 89, 84].

DeWitt, in a slightly different setup, addressed the gravitational drag effect on a superconductor [45]. He considered a (thick) superconducting ring at rest and an axially symmetric mass placed in its centre. This mass is set in rotation, generating a Thirring-Lense field which acts on the superconductor (see [90] for a similar result in covariant form).

He starts from the classical Lagrangian of an electron in electromagnetic and gravitational fields,

$$
L=-m c\left(-g_{\mu \nu} \dot{x}^{\mu} \dot{x}^{\nu}\right)^{1 / 2}+\frac{e}{c} A_{\mu} \dot{x}^{\mu} .
$$

This is a purely classical model and does not capture quantum-mechanical spin effects (cf. Sect. 3.2.3). Using the approximation of weak gravitational fields and small velocities (cf. Sect. 4.1.1, the Hamiltonian 
for the many-electron system reads

$$
H=\sum_{n}\left\{\left(\frac{1}{2 m}\left[\mathbf{p}_{n}-\mathbf{F}\left(x_{n}\right)\right]^{2}+V\left(x_{n}\right)\right\}+V_{\text {int }} .\right.
$$

Here, the generalized magnetic vector potential $\mathbf{F}=\frac{e}{c} \mathbf{A}-\frac{2 m}{c} \mathbf{A}_{\mathrm{g}}$ includes the magnetic as well as the gravitomagnetic potentials. The generalized electric potential is defined by $V=e \Phi-m \Phi_{\mathrm{g}}$. $V_{\text {int }}$ includes the electron-electron potential due to Cooper-pairing of the electrons. It was shown in [91, 92] that in the presence of a gravitational field, the so-called Schiff-Barnhill field $\mathbf{K}=-\nabla V=e \mathbf{E}+m \nabla \Phi_{\mathrm{g}}$, and not $\mathbf{E}$ alone, vanishes in an ordinary conductor. DeWitt argues that if one now applies the BCS formalism to the Hamiltonian (68), the result is that a modified Meissner effect is valid for $e \mathbf{B}-2 m \mathbf{B}_{\mathrm{g}}$ instead of for $\mathbf{B}$ alone, where $\mathbf{B}=\nabla \times \mathbf{A}$ and $\mathbf{B}_{\mathrm{g}}=\nabla \times \mathbf{A}_{\mathrm{g}}$. The same is true for the flux quantization condition, which now reads

$$
\oint_{S}\left(e \mathbf{B}-2 m \mathbf{B}_{\mathrm{g}}\right) \cdot d \mathbf{S}=n \frac{h c}{2} .
$$

If one considers the setup of DeWitt described above, then the condition

$$
\oint_{S}\left(e \mathbf{B}-2 m \mathbf{B}_{\mathrm{g}}\right) \cdot d \mathbf{S}=0
$$

holds for the superconducting ring, in case that no external fields are applied and the mass in the centre is at rest. As soon as the mass inside the ring is rotated, it produces a Thirring-Lense field which acts on the superconductor. Since not $\mathbf{B}$ but the combination $e \mathbf{B}-2 m \mathbf{B}_{\mathrm{g}}$ vanishes, this means that a gravitomagnetic flux induces an electric current in the superconducting ring which could (theoretically) be measured. Using the formula for the Thirring-Lense effect, he makes an estimate of this current (here, SI units are used),

$$
I \sim \epsilon_{0} c^{4} \kappa m M v / e d,
$$

where $\kappa=8 \pi G / c^{4}, m$ is the electron mass, and $M, v$, and $d$ are the mass, rim velocity, and diameter of the rotating body, respectively. Taking the values $M=1 \mathrm{~kg}$ and $v=300 \times 2 \pi \times d[\mathrm{~m}] / 2 \mathrm{~m} / \mathrm{s}$, one obtains $I \approx 10^{-29} \mathrm{~A}$.

Today, SQUID-type measurements are able to measure currents in the order of fA with relative uncertainties in the order of $10^{-3}$ [93 94]. Thus these currents are presently out of experimental reach. It should also be noted that other effects, such as Thirring-Lense fields originating from the Earth, need to be considered in order to isolate the effects caused by the rotating mass.

\subsubsection{Currently proposed experiments}

The idea of Tajmar and de Matos [54, 95] is the combination of two effects: the consideration of the London moment as well as the gravitomagnetic field caused by the rotation of a superconducting mass. To this end, they consider a superconductor rotated about its axis and include gravitational drag effects. Following DeWitt [45] and Ross [90], they alter the quantization conditions to include gravitomagnetic potentials as well. This yields modified London and Ginzburg-Landau quantization conditions. Instead of 63, one now finds ${ }^{10}$

$$
\oint_{C}\left(m \mathbf{v}_{\mathrm{s}}+\frac{e}{c} \mathbf{A}-\frac{2 m}{c} \mathbf{A}_{\mathrm{g}}\right) \cdot \mathrm{d} \mathbf{l}=n \frac{h}{2},
$$

\footnotetext{
10 The additional factor 2 (or 2/c) which appears in the following equations when comparing to the results of [54] arises because of the different definitions of the gravitoelectromagnetic potentials, see Sect. 4.1.1.
} 
while instead of 65) one has

$$
\oint_{S} \mathbf{B} \cdot \mathrm{d} \mathbf{S}-\frac{2 m}{e} \oint_{S} \mathbf{B}_{\mathrm{g}} \cdot \mathrm{d} \mathbf{S}+\frac{m c}{e^{2} n_{\mathrm{S}}} \oint_{C} \mathbf{j} \cdot \mathrm{d} \mathbf{l}+\frac{2 m c}{e} \boldsymbol{\omega} \cdot \mathbf{S}=n \frac{h c}{2 e} .
$$

The inclusion of the gravitomagnetic field should in principle be witnessed in the measurements of the London moment as well as the Cooper pair mass. In analogy to the argumentation used without considering gravitomagnetic fields, Tajmar and de Matos consider the cases of a thick and thin superconducting ring, respectively. For the thick ring, one obtains a modification of the London moment,

$$
\mathbf{B}=-\frac{2 m c}{e} \boldsymbol{\omega}+\frac{2 m}{e} \mathbf{B}_{\mathrm{g}},
$$

and for the thin ring, a modified equation for the Cooper pair mass $m^{\prime}=2 m$ yields

$$
\frac{h}{m^{\prime}}=2 S\left(\Delta \omega+\frac{\Delta B_{\mathrm{g}, \text { lattice }}}{c}\right)
$$

where the additional term arises from the contribution of the superconductor's neutral lattice structure.

According to the classical coupling scheme discussed in Sect. 4.1.2 the gravitomagnetic field would be too small to be detectable. Considering the modified London moment (74) and the coupling equation 59, one obtains

$$
\frac{B}{c} \approx \frac{2 m}{e} \omega, \quad \frac{B_{\mathrm{g}}}{c} \approx G\left(\frac{2 m}{e}\right)^{2} \omega \ll \frac{B}{c} .
$$

This would also imply negligible effects in the measurement of the Cooper pair mass. On the other hand, Tajmar and de Matos [54] suggest that this classical coupling between the two fields might be altered in the interaction of gravitational fields with quantum systems (cf. Sects. 3.2.2 3.2.3), as is the case here. It is argued that the gravitomagnetic field could be responsible for the experimental discrepancies of the measurements of the London moment and the superconducting mass [88, 86], which are correct only up to a few percent.

Assuming that the experimental discrepancies can be fully attributed to the inclusion of gravitomagnetic effects, Tajmar and de Matos find values of $B_{\mathrm{g}} \approx 7,000 \mathrm{c} / 2 \mathrm{~Hz}$ for the measurement of the London moment by Hildebrandt [88] and $B_{\mathrm{g}} \approx 1.65 \times 10^{-5} \mathrm{c} / 2 \mathrm{~Hz}$ for the determination of the Cooper pair mass by Tate [86]. ${ }^{11}$ While these large values do not disqualify the inclusion of the gravitomagnetic effects in the calculations, since the presence of these fields is theoretically expected, it is strongly questionable whether these effects, which are expected to be much smaller than the above values, can be isolated from other systematic errors which arise in these experiments. Furthermore, the calculations in Tate's experiment are very sensitive to the effective mass of the Cooper pairs in Niobium, and thus already the correction of the theoretical mass would lead to better agreement with the experiment.

Finally, we want to mention that the same applies to the experiment conducted on rotating superfluids in [96]. Tajmar and de Matos suggest extending the Josephson current which is measured to include the gravitomagnetic field $\mathbf{B}_{\mathrm{g}}$ [54]; however, the field which would be needed in order to strongly influence the experiment is very large. Again, there is no reason why it should be so based on the present knowledge about the coupling strength between quantum fluids and gravitational fields.

11 Again, the values differ from those found in 54 due to the different definitions for the gravitoelectromagnetic potentials. 


\subsection{Conclusion on proposed experiments with rotating superconductors}

To summarize, we have reviewed the approaches suggested in dealing with rotating superconductors in which weak gravitational fields have been taken into account. An estimate of DeWitt has shown that the induced current in a superconducting ring exposed to Thirring-Lense fields is out of experimental reach today, even if it is possible to isolate the effect caused by the rotating mass from those of the environment. Generally, it can be argued that there is not any theoretical or experimental knowledge as yet that would suggest performing further experiments on rotating superconductors, since any realistic estimates would not explain the proposed experimental discrepancies, for example in Hildebrandt's and Tate's experiments. It would thus be advisable, as already mentioned in Sect. 3.2.5 to first deepen the theoretical knowledge of the coupling between electro- and gravitoelectromagnetic fields.

\section{Summary and conclusion}

In this study, we have discussed possible interactions between two basic foundations of physics: quantum theory on the one hand and the theory of general relativity on the other. The basic ideas and theories were presented and important experiments as well as current propositions mentioned.

Since much research has recently concerned the interaction of gravitational fields with rotating and stationary quantum fluids (quantum systems with macroscopic phase coherence), we have discussed the proposed ideas and evaluated their results. Since gravity couples to all forms of energy, it is clear that coupling occurs, and we want to stress that it is of utmost importance to investigate this interaction further; however, we do not feel that the propositions lead to effects which can presently be measured in the laboratory.

To be more specific, the ideas regarding the generation (and detection) of gravitational waves via quantum systems should be considered theoretically in order to gain a better understanding of the exact coupling strength; only in this way, it is possible to assess the outcome of experiments conducted to this end. To our knowledge, there exists no general non-relativistic derivation of the coupling of an electron to both eletromagnetic and gravitational fields, neither in the post-Newtonian approximation nor in the background of a gravitational wave. Thus, it is not possible to make predictions about the coupling strengths. It is highly recommended that this derivation be performed in order to gain qualitative as well as quantitative results.

Summarizing the propositions for experiments using rotating superconductors, there does not seem to be evidence for the inclusion of the gravitomagnetic fields on experimental grounds. The classical coupling scheme discussed yields interactions which can be neglected. Of course, as a result of GR, the gravitoelectromagnetic fields should be considered in the calculations. Yet, as stated before, we recommend that, even though these effects are interesting in their theoretical origin and should be investigated further, this should be done in a theoretical context to arrive at quantum coupling mechanisms which could lead to experimental results.

\section{Acknowledgements}

This work was supported by the European Space Agency (ESA) under the Ariadna scheme of the Advanced Concepts Team, contract 18152/04/NL/MV. Our thanks go to the ESA, and especially to Andreas Rathke and Clovis de Matos for their comments on this article. We would also like to thank Alexander Altland, Friedrich Hehl, Siddhartha Lal, Bahram Mashhoon, Tobias Micklitz, Thomas Nattermann, Yuri Obukhov, and Bernd Rosenow for helpful discussions and Jürgen Melcher, Gerd-Dietmar Willenberg, and Alexander Zorin from the Physikalisch-Technische Bundesanstalt for their expertise on current measurements. 


\section{References}

[1] C. Kiefer, Quantum gravity (Oxford University Press, Oxford, 2004).

[2] M. M. Nieto and S. G. Turyshev, Finding the origin of the Pioneer anomaly. Class. Quantum Grav., 21, 4005-23 (2004).

[3] G. Börner, The early universe (Springer, Berlin, 2003).

[4] C. M. Will, The confrontation between general relativity and experiment. Living Rev. Relativity, 4, 4 (2001), cited on July 7, 2004.

[5] N. Ashby, Relativity in the global positioning system. Living Rev. Relativity, 6, 1 (2003), cited on July 7, 2004.

[6] O. Bertolami, C. J. de Matos, J. C. Grenouilleau, O. Minster, and S. Volonté, Perspectives in fundamental physics in space. $g r-q C / 0405042$

[7] E. Joos, H.D. Zeh, C. Kiefer, D. Giulini, J. Kupsch, and I.-O. Stamatescu, Decoherence and the appearance of a classical world in quantum theory, 2nd edition (Springer, Berlin, 2003). See also www . decoherence. de.

[8] C. Lämmerzahl, The Einstein equivalence principle and the search for new physics. In: Quantum gravity-from theory to experimental search, edited by D. Giulini, C. Kiefer, and C. Lämmerzahl (Springer, Berlin, 2003), pp. 367-400.

[9] D. N. Page and C. D. Geilker, Indirect evidence of quantum gravity. Phys. Rev. Lett., 47, 979-82 (1981).

[10] C.-I. Kuo and L. H. Ford, Semiclassical gravity theory and quantum fluctuations. Phys. Rev. D, 47, 4510-9 (1993).

[11] S. W. Hawking, Particle creation by black holes. Commun. Math. Phys., 43, 199-220 (1975).

[12] W. G. Unruh, Notes on black-hole evaporation. Phys. Rev. D, 14, 870-92 (1976); P. C. W. Davies, Scalar particle production in Schwarzschild and Rindler metrics. J. Phys. A, 8, 609-16 (1975); S. A. Fulling, Nonuniqueness of canonical field quantization in Riemannian space-time. Phys. Rev. D, 7, 2850-62 (1973).

[13] J. M. Leinaas, Unruh effect in storage rings. In: Quantum aspects of beam physics, edited by P. Chen (World Scientific, Singapore, 2003), pp. 336-52.

[14] C. Barcelo, S. Liberati, and M. Visser, Probing semiclassical analogue gravity in Bose-Einstein condensates with widely tunable interactions. Phys. Rev. A, 68, 053613 (2003).

[15] R. Penrose, Wavefunction collapse as a real gravitational effect. In: Mathematical Physics 2000, edited by A. Fokas et al. (Imperial College Press, London, 2000), pp. 266-82.

[16] W. Marshall, C. Simon, R. Penrose, and D. Bouwmeester, Towards quantum superpositions of a mirror. Phys. Rev. Lett., 91, 130401 (2003).

[17] Y. Fuji and K. Maeda, The scalar-tensor theory of gravitation (Cambridge University Press, Cambridge, 2003).

[18] F. W. Hehl, On the kinematics of the torsion of space-time. Found. Phys., 15, 451-71 (1985).

[19] Y. Ne'eman and F. W. Hehl, Test matter in a spacetime with nonmetricity. Class. Quantum Grav., 14, A251-9 (1997).

[20] J. D. Bjorken and S. D. Drell, Relativistic quantum mechanics (McGraw-Hill, New York, 1964).

[21] C. Lämmerzahl, A Hamilton operator for quantum optics in gravitational fields. Phys. Lett. A, 203, 12-7 (1995).

[22] F. W. Hehl and W.-T. Ni, Inertial effects of a Dirac particle. Phys. Rev. D, 42, 2045-8 (1990).

[23] Y. N. Obukhov, Spin, Gravity, and Inertia. Phys. Rev. Lett., 86, 192-5 (2001).

[24] F. W. Hehl, J. Lemke, and E. W. Mielke, Two lectures on fermions and gravity. In: Geometry and theoretical physics, edited by J. Debrus and A. C. Hirshfeld (Springer, Berlin, 1991), pp. 56-140.

[25] B. Mashhoon, On the coupling of intrinsic spin with the rotation of the Earth. Phys. Lett A, 198, 9-13 (1995).

[26] E. Fischbach, B. S. Freeman, and W.-K. Cheng, General-relativistic effects in hydrogenic systems. Phys. Rev. D, 23, 2157-80 (1981).

[27] C. Lämmerzahl, Constraints on space-time torsion from Hughes-Drever experiments. Phys. Lett. A, 228, 223-31 (1997).

[28] I. L. Shapiro, Physical aspects of the space-time torsion. Phys. Rep., 357, 113-213 (2002).

[29] K. Bongs and K. Sengstock, Physics with coherent matter waves. Rep. Prog. Phys., 67, 907-63 (2004).

[30] R. Colella, A. W. Overhauser, and S. A. Werner, Observation of gravitationally induced quantum interference. Phys. Rev. Lett., 34, 1472-4 (1975).

[31] U. Bonse and T. Wroblewski, Measurement of neutron quantum interference in noninertial frames. Phys. Rev. Lett., 51, 1401-4 (1983).

[32] S. A. Werner, J.-L. Staudenmann, and R. Colella, Effect of Earth's rotation on the quantum mechanical phase of the neutron. Phys. Rev. Lett., 42, 1103-6 (1979).

[33] V. V. Nesvizhevsky et al., Quantum states of neutrons in the Earth's gravitational field. Nature, 415, 297-9 (2002).

[34] C. Lämmerzahl, Quantum tests of the foundations of general relativity. Class. Quantum Grav., 15, 13-27 (1998). 
[35] F. W. Hehl, Y. N. Obukhov, and B. Rosenow, Is the quantum Hall effect influenced by the gravitational field? Phys. Rev. Lett., 93, 096804 (2004).

[36] F. Pinto, Rydberg atoms in curved space-time. Phys. Rev. Lett., 70, 3839-43 (1993); U. Fischer, Transition probabilities for a Rydberg atom in the field of a gravitational wave. Class. Quantum Grav., 11, 463-74 (1994); L. Parker, D. Vollick, and I. Redmount, Atomic spectra in the gravitational field of a collapsing prolate spheroid. Phys. Rev. D, 56, 2113-7 (1997).

[37] R. Y. Chiao and A. D. Speliotopoulos, Towards MIGO, the matter-wave interferometric gravitational-wave observatory, and the intersection of quantum mechanics with general relativity. J. Mod. Opt., 51, 861-99 (2004); R. Y. Chiao and A. D. Speliotopoulos, A crystal-based matter-wave interferometric gravitational-wave observatory. gr-qc/0312100

[38] A. Roura, D. R. Brill, B. L. Hu, and C. W. Misner, Gravitational wave detectors based on matter wave interferometers (MIGO) are no better than laser interferometers (LIGO). gr-qC/0409002

[39] C. Beck and M. C. Mackey, Has dark energy been measured in the lab? astro-ph/0406504

[40] C. W. Misner, K. S. Thorne, and J. A. Wheeler, Gravitation (Freeman, San Francisco, 1973).

[41] N. Straumann, General relativity: with applications to astrophysics (Springer, Berlin, 2004).

[42] R. Wald, General relativity (The University of Chicago Press, Chicago, 1984).

[43] S. Rowan and J. Hough, Gravitational wave detection by interferometry (ground and space), Living Rev. Relativity, 3, 3 (2000), cited on July 7, 2004; The LIGO Scientific Collaboration: B. Abbott, et al., Analysis of LIGO data for gravitational waves from binary neutron stars. Phys. Rev. D, 69, 122001 (2004).

[44] S. Weinberg, Gravitation and cosmology: Principles and applications of the general theory of relativity (Wiley, New York, 1972).

[45] B. S. DeWitt, Superconductors and gravitational drag. Phys. Rev. Lett., 24, 1092-3 (1966).

[46] G. Papini, London moment of rotating superconductors and Lense-Thirring fields of general relativity. Nuovo Cimento B, 45, 66-8 (1966).

[47] G. Papini, Particle wave functions in weak gravitational fields. Nuovo Cimento B, 52, 136-41 (1967).

[48] G. Papini, Detection of inertial effects with superconducting interferometers. Phys. Lett. A, 24, 32-3 (1967).

[49] J. Anandan, Gravitational and inertial effects in quantum fluids. Phys. Rev. Lett., 47, 463-7 (1981); R. Y. Chiao, Interference and inertia: A superfluid-helium interferometer using an internally porous powder and its inertial interactions. Phys. Rev. B, 25, 1655-62 (1982); J. Anandan and R.Y. Chiao, Gravitational radiation antennas using the Sagnac effect. Gen. Rel. Grav., 14, 515-21 (1982); J. Anandan, Gravitational and inertial effects in quantum fluids. Phys. Rev. Lett., 52, 401 (1984).

[50] J. Anandan, Detection of gravitational radiation using superconducting circuits. Phys. Lett. A, 110, 446-50 (1985).

[51] H. Peng and D. G. Torr, The electric field induced by a gravitational wave in a superconductor: A principle for a new gravitational wave antenna. Gen. Rel. Grav., 22, 53-9 (1990); H. Peng, D. G. Torr, E. K. Hu, and B. Peng, Electrodynamics of moving superconductors and superconductors under the influence of external forces. Phys. Rev. B, 43, 2700-4 (1991) .

[52] A. D. Speliotopoulos, Quantum mechanics and linearized gravitational waves. Phys. Rev. D, 51, 1701-9 (1995).

[53] M. Tajmar and C. J. de Matos, Coupling of electromagnetism and gravitation in the weak field approximation. $J$. Theoretics, 3, 1 (2001) .

[54] M. Tajmar and C. J. de Matos, Gravitomagnetic field of a rotating superconductor and of a rotating superfluid. Physica C, 385, 551-4 (2003).

[55] R. Y. Chiao, Conceptual tensions between quantum mechanics and general relativity: Are there experimental consequences? In: Science and ultimate reality: Quantum theory, cosmology and complexity, edited by J. D. Barrow, P. C. Davies, and C. L. Harper (Cambridge University Press, Cambridge, 2003), pp. 254-79.

[56] R. Y. Chiao, Conceptual tensions between quantum mechanics and general relativity: Are there experimental consequences, e.g., superconducting transducers between electromagnetic and gravitational radiation? gr-qc/0208024

[57] R. Y. Chiao, W. J. Fitelson, and A. D. Speliotopoulos, Search for quantum transducers between electromagnetic and gravitational radiation: A measurement of an upper limit on the transducer conversion efficiency of yttrium barium copper oxide. $g r-q c / 0304026$

[58] A. L. Licht, Gravitational waves on conductors. gr-qc/0402109

[59] A. A. Abrikosov, Fundamentals of the theory of metals (Elsevier Science Publishers, Amsterdam, 1988).

[60] J. K. Jain, Composite fermions. In: Perspectives in quantum Hall effects, edited by S. D. Sarma and A. Pinczu (Wiley, New York, 1997), pp. 265-307. 
[61] R. B. Laughlin, Anomalous quantum Hall effect: An incompressible quantum fluid with fractionally charged excitations. Phys. Rev. Lett., 50, 1395-8 (1983).

[62] D. Pines and P. Nozières, The theory of quantum liquids, Vol. 2 (Addison-Wesley, Redwood City, 1990).

[63] C. N. Yang, Concept of off-diagonal long-range order and the quantum phases of liquid He and of superconductors. Rev. Mod. Phys., 34, 694-704 (1962).

[64] M. Born and E. Wolf, Principles of optics (Cambridge University Press, Cambridge, 1999).

[65] V. B. Braginsky, Y. I. Vorontsov, and K. S. Thorne, Quantum nondemolition measurements. Science, 209, 547-57 (1980).

[66] V. B. Braginsky and F. Y. Khalili, Quantum measurement (Cambridge University Press, Cambridge, 1999).

[67] A. Buonanno and Y. Chen, Improving the sensitivity to gravitational-wave sources by modifying the input-output optics of advanced interferometers. Phys. Rev. D, 69, 102004 (2004).

[68] Y. Aharonov and D. Bohm, Significance of electromagnetic potentials in the quantum theory. Phys. Rev., 115, 485-91 (1959).

[69] R. G. Chambers, Shift of an electron interference pattern by enclosed magnetic flux. Phys. Rev. Lett., 5, 3-5 (1960).

[70] M. V. Berry, Quantal phase factors accompanying adiabatic changes. Proc. R. Soc. Lond., A392, $45-57$ (1984).

[71] A. Shapere and F. Wilczek (eds.), Geometric phases in physics, in: Advanced Series in Mathematical Physics, Vol. 5 (World Scientific, Singapore, 1989).

[72] T. Bitter and D. Dubbers, Manifestation of Berry's topological phase in neutron spin rotation. Phys. Rev. Lett., 59, 251-4 (1987).

[73] A. Tomita and R. Y. Chiao, Observation of Berry's topological phase by use of an optical fiber. Phys. Rev. Lett., 57, 937-40 (1986).

[74] G. Delacrétaz, E. R. Grant, R. L. Whetten, L. Wöste, and J. W. Zwanziger, Fractional quantization of molecular pseudorotation in Na3. Phys. Rev. Lett., 56, 2598-601 (1986).

[75] A. Stern, Berry's phase, motive forces, and mesoscopic conductivity. Phys. Rev. Lett., 68, 1022-5 (1992); D. Loss, P. Goldbart, and A. V. Balatsky, Berry's phase and persistent charge and spin currents in textured mesoscopic rings. Phys. Rev. Lett., 65, 1655-8 (1990); D. Loss and P. M. Goldbart, Persistent currents from Berry's phase in mesoscopic systems. Phys. Rev. B, 45, 13544-61 (1992); Y. Lyanda-Geller and P. M. Goldbart, Mesoscopic phenomena in Bose-Einstein systems: Persistent currents, population oscillations, and quantal phases. Phys. Rev. A, 61, 043609 (2000).

[76] E. M. Q. Jariwala, P. Mohanty, M. B. Ketchen, and R. A. Webb, Diamagnetic persistent current in diffusive normal-metal rings. Phys. Rev. Lett., 86, 1594-7 (2001).

[77] J. Lense and H. Thirring, Über den Einfluß der Eigenrotation der Zentralkörper auf die Bewegung der Planeten und Monde nach der Einsteinschen Gravitationstheorie. Phys. Z., 19, 156-63 (1918); English translation: B. Mashhoon, F. W. Hehl, and D. S. Theiss, On the influence of the proper rotation of central bodies on the motions of planets and moons according to Einstein's theory of gravitation. Gen. Rel. Grav., 16, 727-41 (1984).

[78] R. L. Forward, General relativity for the experimentalist. Proc. IRE, 49, 892-904 (1961).

[79] B. Mashhoon, Gravitoelectromagnetism: A brief review. $g r-q c / 0311030$

[80] O. Bertolami and M. Tajmar, Gravity control and possible influence on space propulsion: A scientific study, ESA-CR(P) 4365 (2003).

[81] F. London, Superfluids, Vol. 1 (Wiley, New York, 1950).

[82] B. Cabrera, H. Gutfreund, and W. A. Little, Relativistic mass corrections for rotating superconductors. Phys. Rev. $B, 25,6644-54$ (1982).

[83] R. G. Rystephanick, Electromagnetic fields in rotating superconductors. Am. J. Phys., 44, 647-8 (1976).

[84] A. A. Verheijen, J. M. van Ruitenbeek, R. de Bruyn Ouboter, and L. J. de Jongh, The London moment for high temperature superconductors. Physica B, 165\&166, 1181-2 (1990).

[85] H. Capellmann, Rotating superconductors: Ginzburg-Landau equations. Eur. Phys. J. B, 25, 25-30 (2002).

[86] J. Tate, B. Cabrera, S. B. Felch, and J. T. Anderson, Precise determination of the Cooper-Pair mass. Phys. Rev. Lett., 62, 845-8 (1989); J. Tate, S. B. Felch, and B. Cabrera, Determination of the Cooper-pair mass in niobium. Phys. Rev. B, 42, 7885-93 (1990).

[87] J. Bardeen, L. N. Cooper, and J. R. Schrieffer, Theory of Superconductivity. Phys. Rev., 108, 1175-1204 (1957).

[88] A. F. Hildebrandt, Magnetic field of a rotating superconductor. Phys. Rev. Lett., 12, 190-1 (1964).

[89] M. A. Sanzari, H. L. Cui, and F. Karwacki, The London moment for heavy-fermion superconductors. Appl. Phys. Lett., 68, 3802-4 (1996).

[90] D. K. Ross, The London equations for superconductors in a gravitational field. J. Phys. A: Math Gen, 16, 1331-5 (1983). 
[91] L. I. Schiff and M. V. Barnhill, Gravitation-induced electric field near a metal. Phys. Rev., 151, 1067-71 (1966).

[92] A. J. Dessler, F. C. Michel, H. E. Rorschach, and G. T. Trammel, Gravitationally induced electric fields in conductors. Phys. Rev., 168, 737-43 (1968).

[93] Private communications from Jürgen Melcher, Physikalisch-Technische Bundesanstalt, Braunschweig, Germany.

[94] N. Feltin, L. Devoille, F. Piquemal, S. V. Lotkhov, and A. B. Zorin, Progress in Measurements of a SingleElectron Pump by means of CCC. IEEE Trans. Instrum. Meas., 52, 599-603 (2003).

[95] M. Tajmar and C. J. de Matos, Extended analysis of gravitomagnetic fields in rotating superconductors and superfluids. gr-qc/0406006

[96] R. W. Simmonds, A. Marchenkov, E. Hoskinson, J. C. Davis, and R. E. Packard, Quantum interference of superfluid ${ }^{3}$ He. Nature, 412, 55-8 (2001). 